\title{
CIRCULAR OBJECT DETECTION USING A MODIFIED HOUGH TRANSFORM
}

\author{
MARCIN SMEREKA, IGNACY DULĘBA \\ Institute of Computer Engineering, Control and Robotics \\ Wrocław University of Technology \\ ul. Janiszewskiego 11/17 \\ 50-372 Wrocław, Poland \\ e-mail: marcin.smereka@pwr.wroc.pl
}

\begin{abstract}
A practical modification of the Hough transform is proposed that improves the detection of low-contrast circular objects. The original circular Hough transform and its numerous modifications are discussed and compared in order to improve both the efficiency and computational complexity of the algorithm. Medical images are selected to verify the algorithm. In particular, the algorithm is applied to localize cell nuclei of cytological smears visualized using a phase contrast microscope.
\end{abstract}

Keywords: Hough transform, medical imaging, image processing, circular shape recognition.

\section{Introduction}

In many practical situations on the factory floor or in nature sciences, a task of the detection of circular objects is encountered. In technical reality, rolling elements are of great usability, while in nature sciences, biological objects are usually circular as they optimize the area-to-perimeter ratio. Two problems are major concern here: either the objects searched for are difficult to distinguish or the detection is to be performed in real time. The first problem is due to non-even illumination, low-contrast, noise or restricted, partial visibility of objects. The second problem is caused by technological requirements (on-line detection, classification and sorting). Real, industry-sourced images of circular objects are frequently well structured as a camera observes them in a particular place of a conveyor belt. Moreover, in this case, an ideal shape of objects to be detected is known in advance. In this paper, a slightly more involved task is considered when real images are sourced from a microscope observing a medical slide. Obviously, techniques developed for medical images can be applied to images rooted in industry as well with some domain-specific modifications.

The joint research conducted at the Wrocław University of Technology and in the Gynecology Clinic GMW in Opole is aimed at the recognition and classification of objects present in phase contrast $(\mathrm{PhC})$ cytological images. PhC microscopy is a modern and promising technology to visualize cellular structures of tissues and smears
(Glab et al., 2001). Contrary to the Papanicolau technique (World Health Organization, 1988), commonly used in medical procedures, structures are visualized without staining and fixing. Therefore, the $\mathrm{PhC}$ technique is suitable to support any immediate diagnosis technique (it can be easily coupled with colposcopy). The most important objects in Ph images are cell nuclei as they carry out valuable information on pre-cancerous cell stages. To construct an effective algorithm of cell detection, as much as possible a-priori knowlegde should be incorporated into the algorithm. It is well known (Ross, 1967) that due to hallo and shading-off effects $\mathrm{Ph}$ microscopy emphasizes object edges. Thus, searching for nuclei can be replaced by searching for their edges. Moreover, for most types of cells their nucleus shape is circular or elliptic, so oval patterns are of main interest. When a fixed magnification microscope is in use, only objects within a prescribed range of radii are considered as potential cell nuclei.

Popular methods of detecting circular or elliptic shapes from low-contrast noisy images are the Circular Hough Transform (CHT) and Elliptical Hough Transform (EHT). In this paper, practical modifications of the CHT are presented in order to improve the sensitivity and specificity of the segmentation of medical microscopic images. This paper is organized as follows: Section 2 presents an overview of methods designed to detect oval shapes. Section 3 describes the original and the modified CHT. In Section 4 different versions of the CHT are compared experimentally. Final conclusions are collected in Section 5 


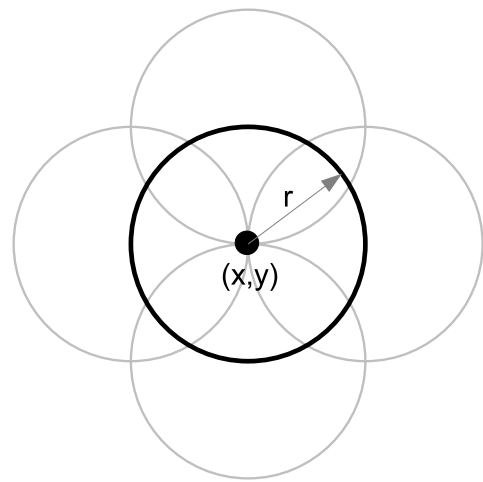

(a)

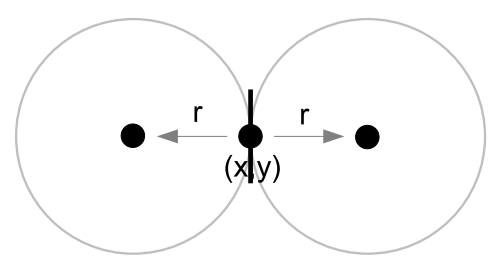

(c)

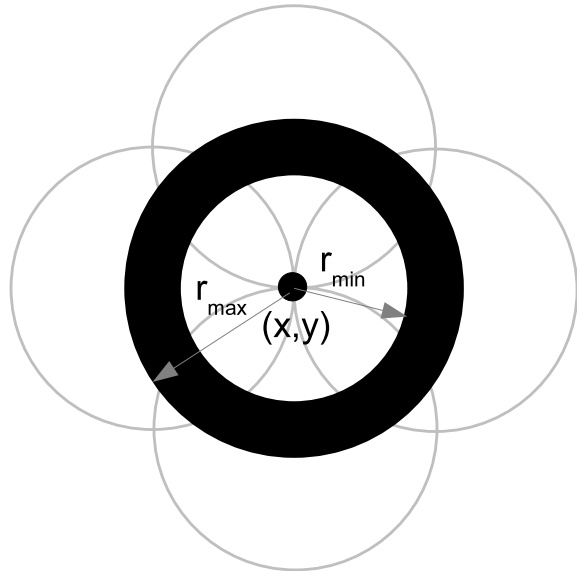

(b)

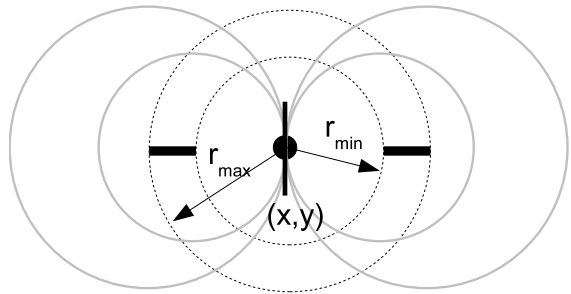

(d)

Fig. 1. Idea of voting: (a) classical CHT, (b) CHT with multiple radii in one parameter space, (c) oriented CHT, (d) oriented CHT with multpile radii in one parameter space.

\section{Related methods}

The detection of circular and elliptic shapes is a common task in computer vision and image recognition. Some methods rely on converting gray-scale images to binary ones using edge detection techniques and calculating numerical shape descriptors. Peura and Ilvarinen (1997) studied some simple shape descriptors. One of them, known as elliptic variance, is especially useful for detecting ellipses. Rosin proposed other descriptors (moment invariants, Euclidean distances) that can be adapted to measure the ellipticity of shapes (Rosin, 2000). Fitzgibbon et al. (1999) were the first to present a direct method for fitting ellipses to a set of points in the least-squares sense. Their method is exploited in the segmentation algorithm presented in this work. Previous methods used a generic conic fitting or an iterative approach to recover elliptic solutions. A variety of 'fit-to-data' functions were discussed in (Rosin, 1996).

Low level edge detection operators do not guarantee continuous boundaries of objects. This makes many image analysis tasks difficult, especially for noisy images. The aim of contour grouping algorithms is to connect edges that are supposed to be sub-parts of the same object. Contour grouping techniques were concentrated mainly on detecting salient curves (Shashua and Ullman, 1991; Zhu, Payne and Riordan, 1996). Their improvements favor closed ( Elder and Zucker, 1996) shapes rather than long and smooth ones.

Methods based on active contours (snakes) are designed to detect objects with boundaries not necessarily defined by gradients (Chan and Vese, 2001). Their basic idea is to reshape an initial curve prvided by any rough segmentation technique, subject to constraints from a given image. Initially, the current curve is set to the initial one. In an iterative process it is moved according to an appropriately designed energy function until eventually it stops in a local optimum. Its final shape is assumed to define the object boundary. Ray et al. (2002) showed that active contours can also be employed for tracking moving objects. The energy function calculates the difference between object features and background features, so it is useful for stained smears. In the case of Ph images, it is difficult to define features that distinguish nuclei and a cytoplasm clearly.

\section{CHT and its variants}

The Hough Transform (HT) has been recognized as a very powerful tool for the detection of parametric curves in 
images ( Duda and Hart, 1972; Hough, 1962). It implements a voting process that maps image edge points into manifolds in an appropriately defined parameter space. Peaks in the space correspond to the parameters of detected curves. The Circle Hough Transform (CHT, Fig. 11a)) is designed to find a circle characterized by a center point $\left(x_{0}, y_{0}\right)$ and a radius $r$, while the Ellipse Hough Transform (EHT) finds elliptical formations coded by a center $\left(x_{0}, y_{0}\right)$, the orientation of the ellipse $\Theta$ and its semiaxes $a$ and $b$. HT algorithms used to detect circles and ellipses are computationally more expensive than line detection algorithms, due to the large number of parameters involved in describing the shapes. To determine a circle, it is necessary to accumulate votes in the three-dimensional parameter space $\left(x_{0}, y_{0}, r\right)$. When detecting an ellipse, the search must be performed in the five-dimensional parameter space $\left(x_{0}, y_{0}, \Theta, a, b\right)$.

To keep computational and memory requirements at an acceptable level, a few improvements of the CTH algorithm were proposed in the image-processing literature. Kimme et al. (1975) proposed to exploit an edge orientation. Accordingly, the point of the image space votes for two points in the parameter space, rather than for a circle (Fig. 1(c)). Minor and Sklansky ( Gerig and Klein, 1986; Minor and Sklansky, 1981) used the same parameter space for different radii (Fig. 1 b) and (d)). Some researchers investigated real-values of radii and considered a complex parameter space to code their values (Atherton and Kerbyson, 1993; Atherton and Kerbyson, 1993). Guil and Zapata (1997) determined circles and ellipses via consecutive determination of their midpoint, orientation and axes. Hierarchical (coarse-to-fine) search techniques successively narrow down the region of interest in images. Consequently, the detailed detection is conducted only in those regions where the coarse detection succeeded (Atiquzzaman, 1999). Competitive implementation of the HT (HT convolution filters, recognition by invariance kernels) is an alternative approach to search in a parameter space (Atherton and Kerbyson, 1999; McLaughlin and Alder, 1998). Some architectures were proposed for parallel computing performed on multiprocessors. They can substantially decrease the time of detection without reducing its computational complexity (Kavianpour et al., 1994).

In our approach, to prepare a phase contrast cytological image for segmentation, an initial image was transformed to an edge image (Fig. 2). Then, to detect a gradient and an orientation for each point, the initial image $I_{1}$ was convolved with $3 \times 3$ horizontal and vertical Sobel operators. Consequently, horizontal and vertical gradient images $I_{X}$ and $I_{Y}$ were obtained. And, finally, to compute the amplitude of an edge gradient and an orientation, the images $I_{X}$ and $I_{Y}$ were transformed into images $I_{A}$ and $I_{\phi}$ expressed in polar coordinates:

$$
\begin{aligned}
& I_{A}^{(x, y)}=\sqrt{\left(I_{X}^{(x, y)}\right)^{2}+\left(I_{Y}^{(x, y)}\right)^{2}}, \\
& I_{\varphi}^{(x, y)}=\operatorname{atan} 2\left(I_{Y}^{(x, y)}, I_{X}^{(x, y)}\right) .
\end{aligned}
$$

For clarity of presentation, in all formulas the normalization transformation was ommited. To separate edges from the background and eliminate very weak edges, thresholding with a constant threshold was performed in the image $I_{A}$.

All the above-mentioned modifications of the CHT were designed either to improve robustness or to simplify computations. The CHT is more robust when hardly visible circles can be detected and noise in an image does not influence the performance of the detection. In the case of phase-contrast medical images, two main problems must be addressed. The first results from object irregularity. Therefore, the uncertainty of radii and orientations must be incorporated into the voting process. The second one is the fact that circular objects (for example, nuclei) can be easily mixed with circular arcs that come from cell walls or other irregular structures. In order to deal with the first problem, a point in the image space can vote for an annular sector in the parameter space, rather than for a

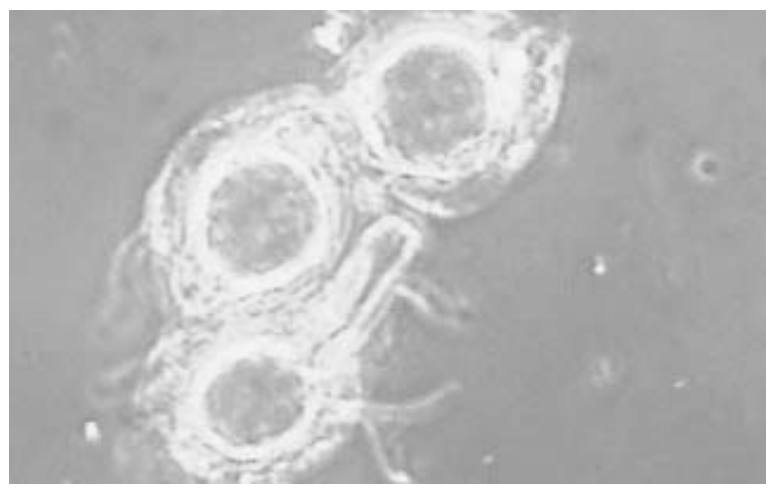

(a)

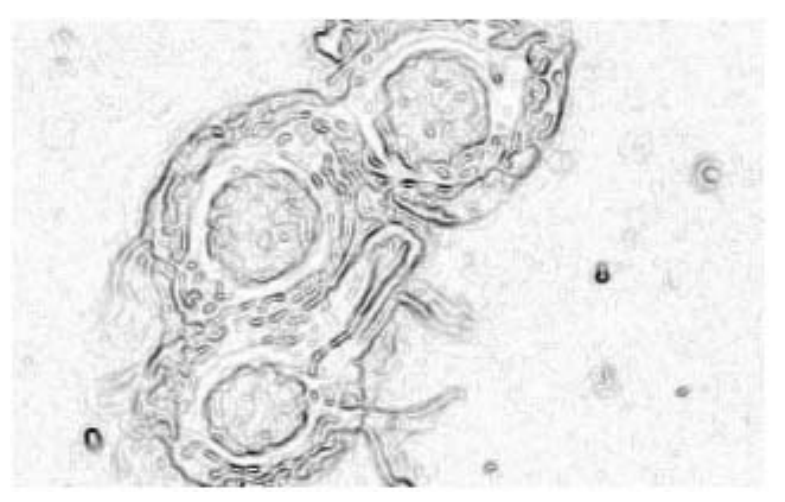

(b)

Fig. 2. Example of phase-contrast images: (a) initial image $I_{1}$, (b) edge amplitude image $I_{A}$. 
single point (cf. Fig. 3 a)). The width of the annulus corresponds to the assumed uncertainty of radii $\left(r_{\min }, r_{\max }\right)$ while the angle of the sector $\alpha$ corresponds to the uncertainty of orientation. Both the parameters can be adjusted experimentally and depend on the magnification of a phase-contrast microscope.

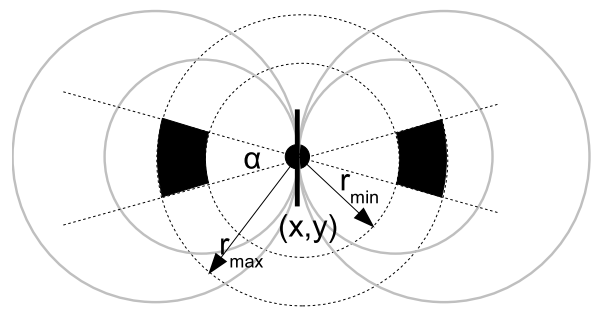

(a)

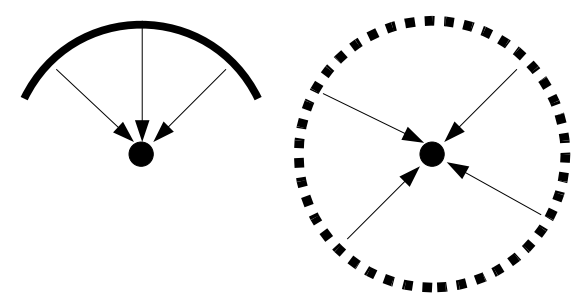

(b)

Fig. 3. (a) Idea of voting with uncertainty handling, the radius $\left[r_{\min }, r_{\max }\right]$ and the orientation $\alpha$, (b) voting from an arc and a circle.

Another original modification of the CHT was designed to distinguish circles from arcs. It should prefer full circles, rather than circular arcs (Fig. 3 b)). Let us divide the full angle into $K$ equi length angular sectors and define the three-dimensional parameter space $\left(x_{0}, y_{0}, k\right)$ where the parameters $\left(x_{0}, y_{0}\right)$ correspond to the coordinates of a midpoint while the third parameter is responsible for the sector label. In each elementary voting process for a fixed point, extra information, based on edge orientation, is used to calculate the angular sector the vote comes from. Then accumulation is performed separately for each sector. After all the votes were counted and the desribed transformation $H\left(x_{0}, y_{0}, k\right)$ was completed, the third dimension of the parameter space was flattened according to the following formula:

$$
H_{1}(x, y)=\prod_{k=1}^{K} \begin{cases}1 & \text { if } H(x, y, k)>0 \\ 0 & \text { otherwise }\end{cases}
$$

The value of 1 in the parameter space $H_{1}\left(x_{0}, y_{0}\right)$ was assigned to those points with a nonzero response in all $K$ sectors. Equation (2) allowed us to segment these places in the parameter space which were likely the centers of circles and to clear locations where the accummulation resulted from arcs or noise. Finally, the $H_{1}$ space contained the centers of circles with the radii range $\left(r_{\min }, r_{\max }\right)$. In the case of cytological images it is very likely that these circles are cell nuclei. Obviously, when $K$ is relatively large, the condition (2) should not be so restrictive and a majority of votes coming from dispersed sectors are enough to classify an object as a circle.

\section{Experiments}

The aim of the first test was to detect circular objects from Fig. 2 assuming that the radius of a cell may vary between 15 and 25 pixels. The image was transformed using different versions of the CHT in order to search for three nuclei visualized in the image. The classical CHT was unable to perform the task as it works only with a constant value of the radius.

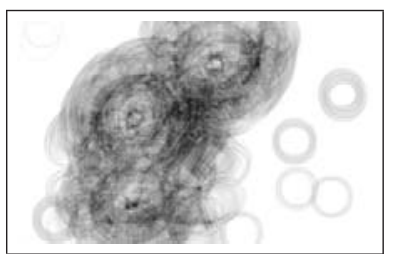

(a)

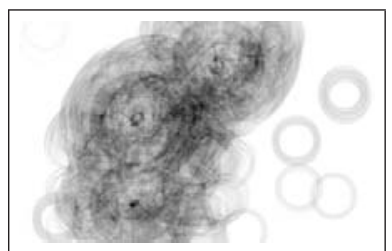

(c)

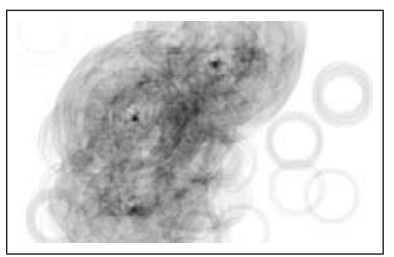

(e)

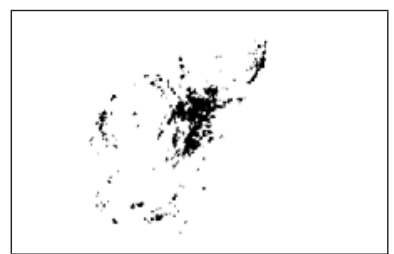

(b)

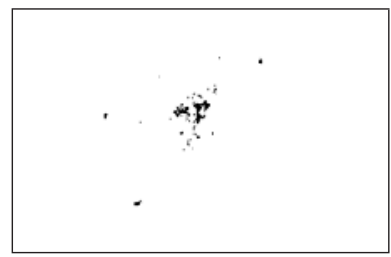

(d)

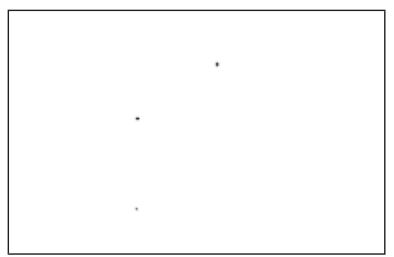

(f)
Fig. 4. Classical CHT for the following radii: (a) 17, (c) 20, (e) 23. The result of thresholding left panel images with the best possible thresholds: b) 86 , d) 64 , f) 36 , respectively.

In Figs. 4(a), (c) and (e), examples of the classical CHT were presented for a fixed cell nucleous radius with the values of $17,20,23$ pixels. To facilitate visualization, the parameter space was normalized to a gray scale and all values are within the range of $[0,255]$. In Figs. 4(b), (d) and (f), the results of the tresholding of the parameter space are presented with a manually adjusted threshold value which was assessed as best for segmentation. For the radii 17 and 20 there was no threshold value capable 
of segmenting the three visible nuclei and, simulataneusly, to neglect the background. For the radius 23 and the threshold value between 31 and 36 , the segmentation was successful. It appears that the classical CHT is effective only in the case when the values of the exact radius and the threshold are known in advance. Unfortunately, it is rather a rare case in real applications. In Fig. 5] another

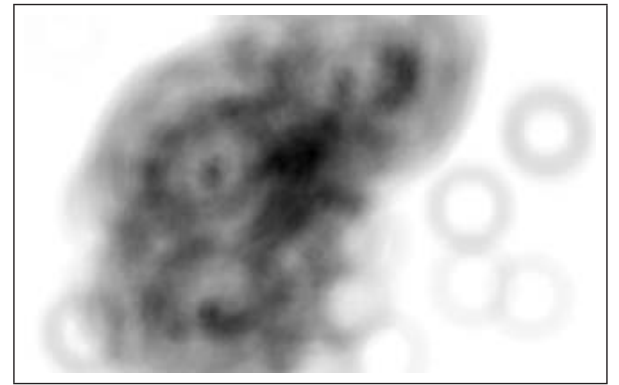

(a)

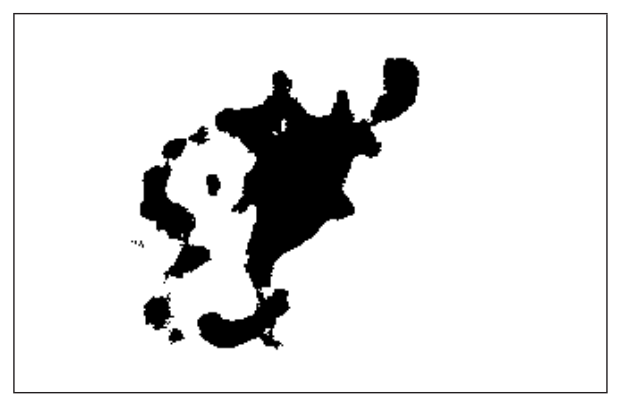

(b)

Fig. 5. (a) CHT with one parameter space for radii between 15 and 25 , (b) result of thresholding with the value of 100

example of the CHT is presented, this time with one parameter space for all radii within the range of 15 to 25 pixels. It corresponds to the idea of voting from Fig. 1(b). As can be seen, also in this case there was no threshold value to detect the three nuclei as the accumulation resulting from the noise was much stronger than the accumulation from circular structures. This type of CHT may be applied to low-noise images only.

In the next experiment, the oriented CHT was examined, which corresponds to the idea visualized in Fig. 1 (c). The oriented CHT was carried out for the radii 17,20 and 23 separately and the results are collected in Figs. 6.b), (d) and (f). Thresholding in the parameter space brought satisfactory results only for $r=23$, where the threshold values between 92 and 100 allowed us to segment the nuclei. Still the results of segmentation were unsatisfactory, this time due to irregularities of the radius and orientation, which were not handled properly by the fixed radius oriented CHT.

Next, the performance of the oriented CHT with a condensed, multi-radii parameter space was examined (its

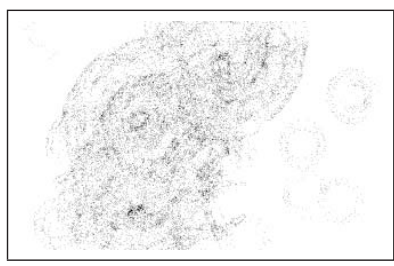

(a)

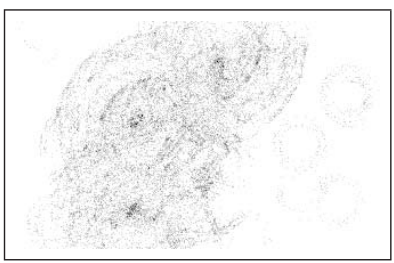

(c)

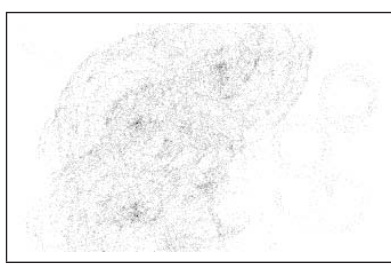

(e)

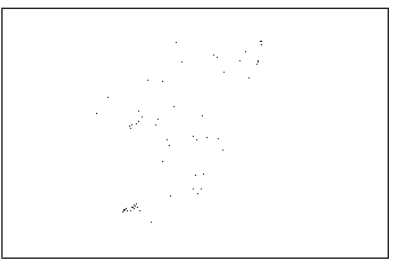

(b)

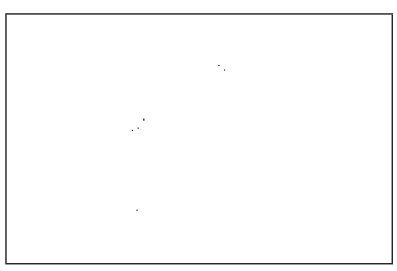

(d)

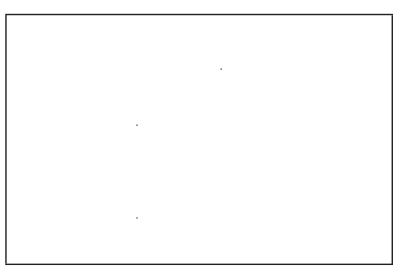

(f)
Fig. 6. Oriented CHT for the radii: (a) 17, (c) 20, (e) 23. The result of thresholding left panel images with the best possible thresholds: (b) 95, (d) 72, (f) 100, respectively.

idea is illustrated in Fig. 1(d)). The results are provided in Fig. 7 for the parameter space covering radii between 15 and 25 pixels. This approach strenghtened midpoints of nuclei, which was also confirmed by thresholding the parameter space. For distant threshold values (between 44 and 77) all nuclei were determined and clearly differed from the background. These results seemed to be promising.

Even better results of segmentation were observed for the oriented CHT with uncertainty included both in the radius and orientation (cf. Fig. 3 (a)). The range of threshold values resulting in a satisfactory segmentation varied between 40 and 92 , which was the widest range for all previously tested methods. In Fig. 8 this version of the CHT is illustrated.

The last experiment illustrated the original modification introduced in this paper. In Fig. 9 the results of the CHT with separate accumulation for $K=8,16$ and 32 sectors are presented. An appropriately adjusted number of sectors $K$ allows us to neglect the stage of thresholding in the parameter space (thus, to choose the threshold value). Locations where the accumulation took the value of 0 for at least one sector were zeroed in the final image (the white background in the figures). Due to the radius and orientation uncertainty handling, the transform appeared to be robust for irregularities and, additionally, resi- 


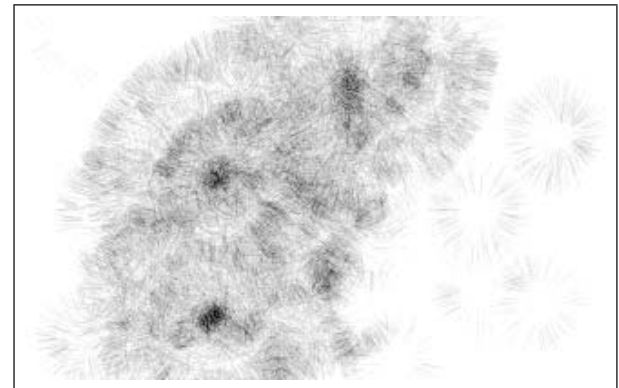

(a)

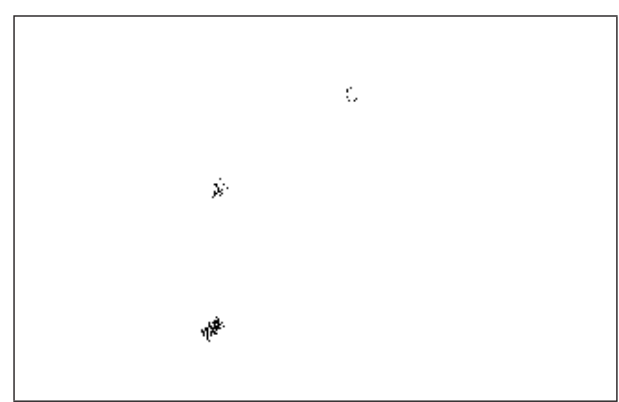

(b)

Fig. 7. (a) Oriented CHT with one parameter space for radii between 15 and 25, (b) thresholding the resulting image with the value of 77 .

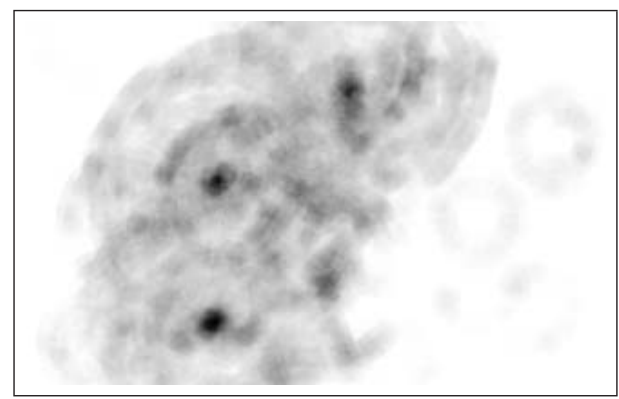

(a)

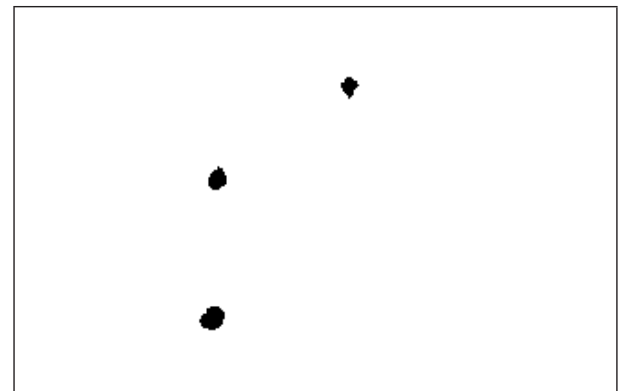

(b)

Fig. 8. (a) Oriented CHT with radius in the range of $[15,25]$ and uncertainty in the orientation $\alpha=20^{\circ}$, (b) result of thresholding with the value of 92 .

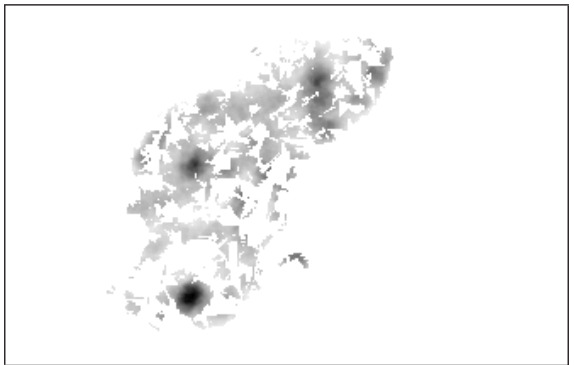

(a)

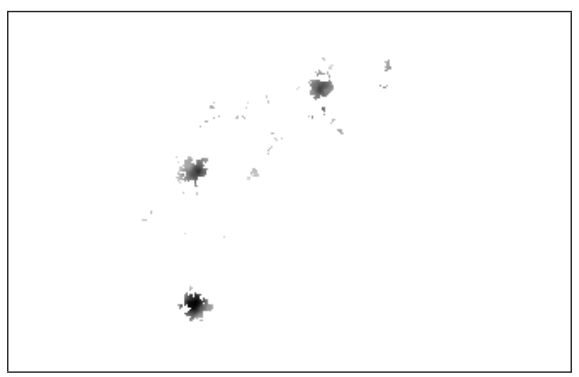

(b)

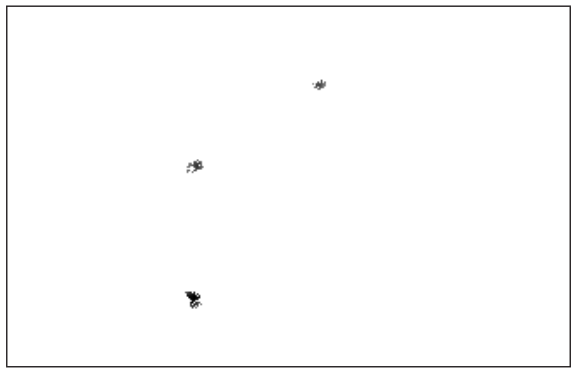

(c)

Fig. 9. Oriented CHT with uncertainty in the radius and orientation $r \in[15,25], \alpha=20^{\circ}$ with separate accumulation for (a) $K=8$, (b) $K=16$, (c) $K=32$ sectors.

stant to the presence of circular arcs in images. Further experiments conducted on other cytological images proved that it was much easier to select a constant number of sectors $K$, rather than the threshold value in the parameter space. This modification of the CHT method was successively employed for the segmentation of cytological images of uterine cervix in order to detect pathological changes (Smereka, 2003).

The computational complexity of the CHT is proportional to the image size and the accummulation area that corresponds to each pixel. Processing a single $640 \times 480$ image took less than one second on an $1.8 \mathrm{GHz}$ Athlon processor with a $512 \mathrm{MB}$ RAM for all examined variants of the CHT.

A statistical comparison of the proposed method with known modifications of the CHT was not performed due to the impossibility of adjusting a common threshold 
value for a series of images. A clear advantage of the new CHT method is the lack of the necessity to select the threshold value at all.

\section{Conclusions}

An effective method for circular object recognition was introduced. This approach was verified on microscopic medical images to recognize nuclei of cells that come from phase contrast microscopy. It is robust for irregularities in detected objects and for disturbances like noise or even lenghty circular arcs. Despite the threedimensional parameter space, the computational complexity corresponds to a two-dimensional HT, because each pixel of the image is assigned to one sector only. The method designed for the detection of demanding (noisy, not clearly distiguishable) circular objects can be applied, after fixing some data with domain-specific values, to any real industry images.

\section{References}

Atherton T. J. and Kerbyson D. J. (1993). The coherent circle Hough transform, Proceedings of the British Machine Vision Conference, Guildford, UK, pp. 269-278.

Atherton T. J. and Kerbyson D. J. (1993). Using phase to represent radius in the coherent circle Hough transform, IEE Coloquium on the Hough Transform 5: 1-4.

Atherton T. J. and Kerbyson D. J. (1999). Size invariant circle detection, Image and Vision Computing 17(11):795-803.

Atiquzzaman M. (1999). Coarse-to-fine search technique to detect circles in images, International Journal of Advanced Manufacture Technologies 15(2): 96-102.

Chan T. F. and Vese L. A. (2001). Active contours without edges, IEEE Transactions on Image Processing 10(2): 266-277.

Duda R. O. and Hart P. E. (1972). Use of the Hough transform to detect lines and curves in pictures, Communications of the ACM 15(1): 11-15.

Elder J. H. and Zucker S. W. (1996). Computing contour closure, Proceedings of the 4-th European Conference on Computer Vision-Volume I, Cambridge, UK, 1, 399-412.

Fitzgibbon A., Pilu M. and Fisher R. B. (1999). Direct least square fitting of ellipses, IEEE Transactions on Pattern Analysis and Machine Inteligence 21(5): 477-480.

Gerig G. and Klein F. (1986). Fast contour identification through efficient Hough transform and simplified interpretation strategy, Proceedings of the International Joint Conference on Pattern Recognition, Paris, France, pp. 498-500.

Glab G., Florczak K., Jaronski J., and Licznerski T. (2001). Gynecological Cyto-diagnosis in Phase Contrast Microscopy, Blackhorse, Warsaw (in Polish).
Guil N. and Zapata E. L. (1997). Low order circle and ellipse Hough transform, Journal of Pattern Recognition 30(10): 1729-1744.

Hough P. V. C. (1962). Methods and means for recognizing complex patterns, U.S. Patent 3; 069; 654.

Kavianpour A., Shoari S. and Bagherzadeh N. (1994). A new approach for circle detection on multiprocessors, Journal of Parallel and Distributed Computing 20(2): 256-260.

Kimme C., Ballard D. and Sklansky J. (1975). Finding circles by an array of accumulators, Communitations of the ACM 18(2): 120-122.

McLaughlin R. A. and Alder M. D. (1998). Technical reportThe Hough transform versus the upwrite, IEEE Transactions on Pattern Analysis and Machine Intelligence 20(4): 396-400.

Minor L. G. and Sklansky J. (1981). Detection and segmentation of blobs in infrared images, IEEE Transactions on Systems, Man, and Cybernetics 11(3): 194-201.

Peura M. and Iivarinen J. (1997). Efficiency of simple shape descriptors, Aspects of Visual Form, Proceedings of the 3rd International Workshop on Visual Forum, Capri, Italy, pp. 443-451.

Ray N., Acton S. T. and Ley K. F. (2002). Tracking leukocytes in vivo with shape and size constrained active contours, IEEE Transactions on Medical Imaging 21(10): 1222-1235.

Rosin P. (1996). Assessing error of fit functions for ellipses, Graphical Models and Image Processing 58(5): 494-502.

Rosin P. (2000). Measuring shape: Ellipticity, rectangularity, and triangularity, Proceedings of the 15-th International Conference on Pattern Recognition, Barcelona, Spain, pp. 1952-1955.

Ross K. F. A. (1967). Phase Contrast and Interference Microscopy for Cell Biologists, Edward Arnold Publishers, London.

Shashua A. and Ullman S. (1991). Grouping contours by iterated pairing network, Neural Info 3: 335-341.

Smereka M. (2003). Nuclei recognition in phase contrast microscopy images, Proceedings of the 3-rd International Conference on Computer Recognition Systems KOSYR, Mitków, Poland, pp. 35-40.

World Health Organization. (1988). Cytological Screening in the Control of Cervical Cancer: Technical Guidelines, World Health Organization, Geneva.

Zhu Q., Payne M. and Riordan V. (1996). Edge linking by a directional potential functions, Image and Vision Computing 14(1): 59-70. 
